Hydroécol. Appl. (2001) Tome 13 Vol. 1, pp. 21-35

\title{
Modélisation écologique tridimensionnelle (3D) de la baie de Seine (Manche, France)
}

\section{Three dimensional (3D) ecological modelling of the "baie de Seine" (English Channel, France)}

\section{P. Cugier, A. Ménesguen, J.-F. Guillaud}

IFREMER, Direction de l'Environnement Littoral, Département d'Ecologie Cótière, B.P. 70, 29280 Plouzané.

Email : pcugier@ifremer.fr

Résumé. - Une modélisation écologique tri-dimensionelle (3D) a été développée en baie de Seine. Elle est constituée d'un modèle 3D hydrosédimentaire (SiAM3D) couplé à un modèle écologique simulant le cycle des éléments nutritifs (azote, phosphore, silicium) et deux classes phytoplanctoniques (diatomées et flagellés). Après validation par confrontation des simulations avec neuf années de mesures, le modèle a été utilisé afin de mieux comprendre le fonctionnement de l'écosystème. II a notamment permis de montrer le rôle de la proportion de silice par rapport à l'azote et au phosphore dans les apports estivaux de la Seine sur la présence plus ou moins forte de flagellés dans le panache du fleuve. Le modèle a également été utilisé pour tester différentes hypothèses de réduction des apports d'azote par la Seine et leurs conséquences sur la production phytoplanctonique dans le panache.

Mots-clés. - modèle 3D, éléments nutritifs, diatomées, flagellés, baie de Seine.

\begin{abstract}
A three dimensional (3D) ecological model is developed and applied to the "baie de Seine " area. It consists of a 3D model for hydrodynamics and sediment transport (SiAM3D) coupled to an ecological model simulating nutrient cycles (nitrogen, phosphorus, silicon) and two phytoplanktonic classes (diatoms and flagellates). The model is validated by confronting simulations with nine year long series of measurements and then used to better understand the ecosystem functioning. It especially allows to show the role of the proportion of silicon with regard to nitrogen and phosphorus in the summer loadings of the Seine river on the more or less strong presence of flagellates in the river plume. The model was also used to test various hypotheses of reduction of nitrogen loading from the Seine river and their consequences on the phytoplanktonic production in the plume.
\end{abstract}

Key words. - 3D model, nutrients, diatoms, flagellates, baie de Seine. 


\section{INTRODUCTION}

L'enrichissement des zones côtières en nutriments, lié aux apports continentaux des fleuves, confère à ces zones une forte capacité de production biologique, allant jusqu'à l'eutrophisation. Ainsi, de fortes poussées phytoplanctoniques sont régulièrement observées dans les panaches de dilution des fleuves. La baie de Seine fait partie de ces zones eutrophisées. L'apport principal dans la baie est celui de la Seine dans la partie Est, dont les débits varient de $100 \mathrm{~m} / \mathrm{s}$ en étiage à $2000 \mathrm{~m} / \mathrm{s}$ en crue. II existe d'autres apports plus faibles comme l'Orne sur la côte sud ou la Vire et la Douve en baie des Veys (fig. 1).

Les apports par la Seine d'azote total, de phosphore et de silice entre
1984 et 1991, pour un débit moyen de $413 \mathrm{~m} / \mathrm{s}$, ont été estimés respectivement à 90700 tonnes/an, 46300 tonnes/an et 10850 tonnes/an (Guillaud, 1993). Au cours des 20 dernières années, les apports d'azote ont par ailleurs progressé de $2 \%$ par an, principalement en raison de l'augmentation des apports de nitrate d'origine agricole (fig. 2).

La baie de Seine présente ainsi de fortes biomasses algales avec des maximums de chlorophylle qui atteignent régulièrement $40 \mu \mathrm{g} / \mathrm{l}$ et qui peuvent parfois dépasser les $50 \mu \mathrm{g} / \mathrm{l}$. De plus, depuis le début des années 80, l'apparition du dinoflagellé toxique Dinophysis cf. acuminata entraîne régulièrement des interdictions de ramassage des coquillages au cours de l'été, et une surveillance estivale de la composition des popu-

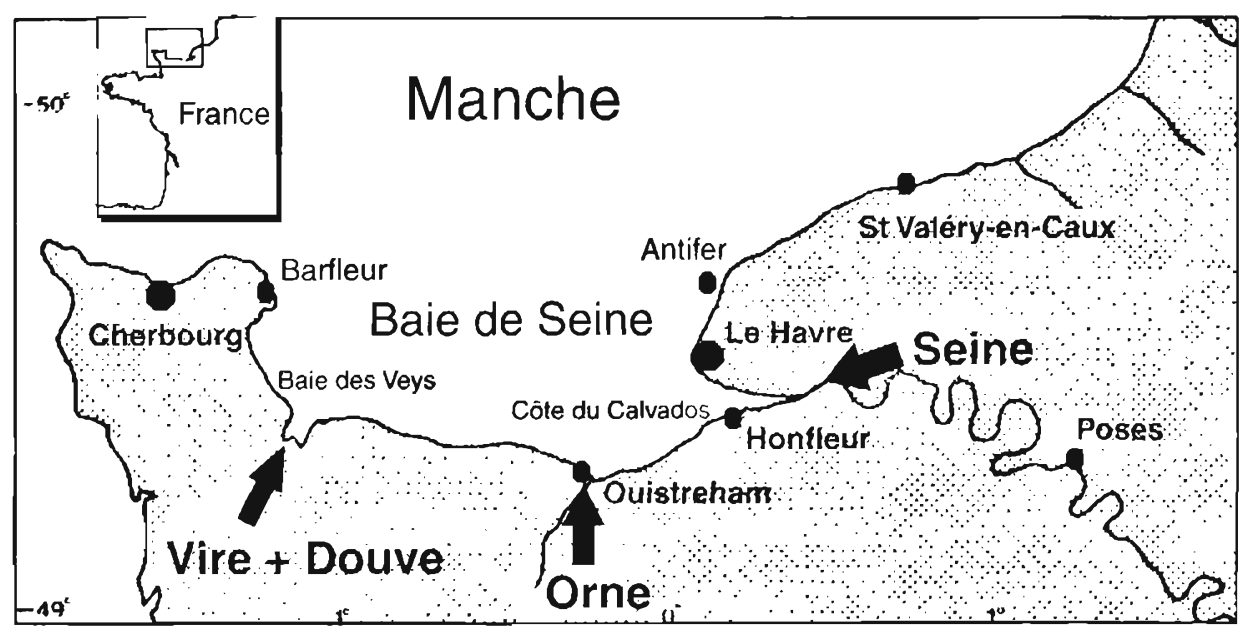

Fig. 1. - Localısatıon de la zone d'étude.

Fig. 1. - Location of the study area. 

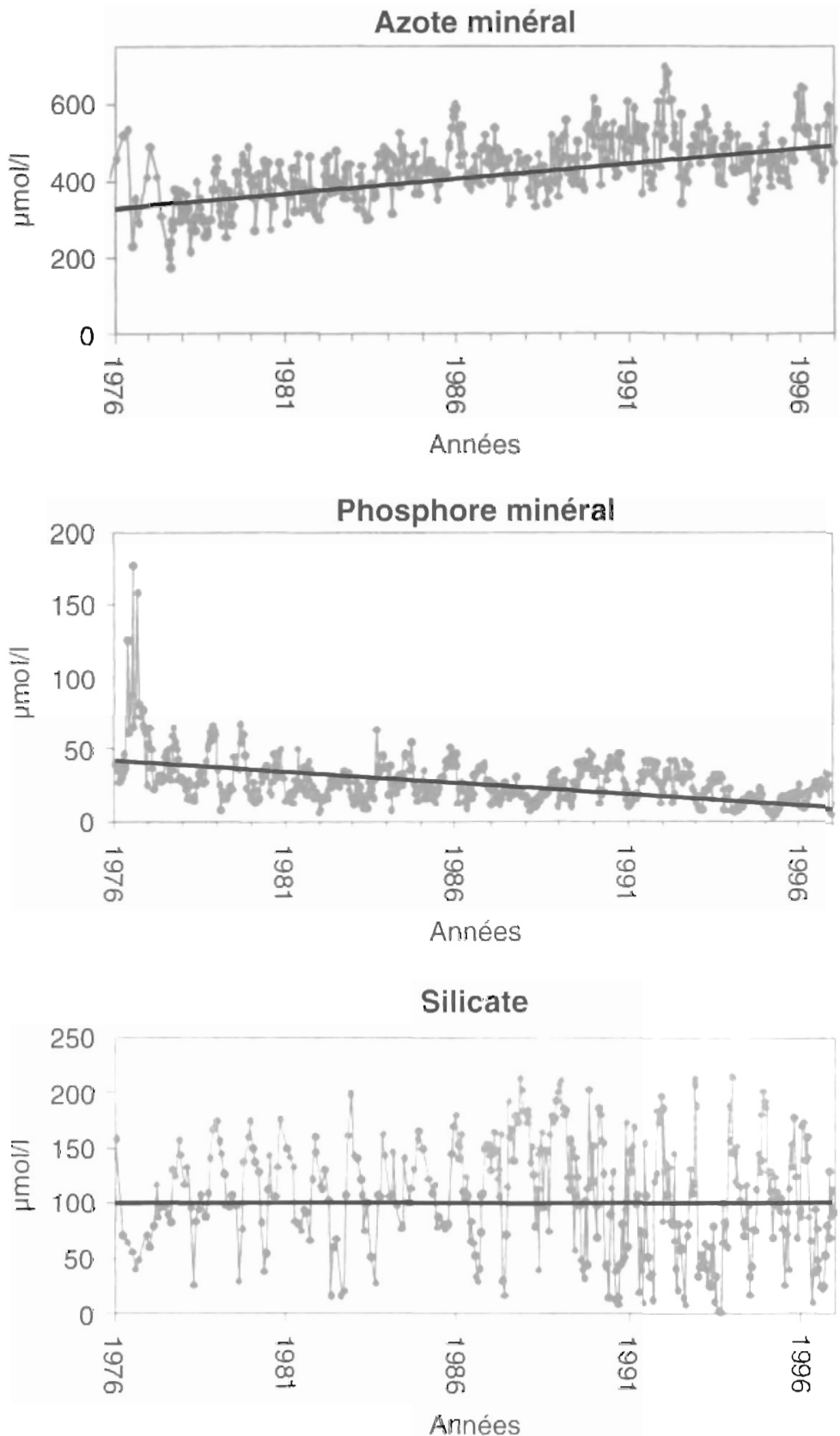

Fig. 2. - Evolution des apports en éléments nutritifs dissous par la Seine entre 1976 et 1996.

Fig. 2. - Evolution of dissolved nutrient loadings from the Seine river between 1976 and 1996. 
lations phytoplanctoniques est nécessaire.

Pour étudier l'impact de ces apports massifs sur la production phytoplanctonique, plusieurs modélisations mathématiques ont été développées (Ménesguen et al., 1995 ; Guillaud et Ménesguen, 1998 ; Guillaud et al., 2000). Ces modèles sont basés sur une spatialisation en compartiments entre lesquels les mouvements horizontaux des masses d'eau sont représentés par des flux advectifs résiduels de marée et des flux dispersifs représentant le mélange turbulent. Ces modèles compartimentaux ont permis notamment d'identifier le rôle respectif de chacun des éléments nutritifs dans la croissance phytoplanctonique. Ainsi, l'effet limitant du phosphore au printemps puis de la silice en été sur la croissance des diatomées, dans le panache, a clairement été mis en évidence.

Afin de mieux représenter l'espace et la dynamique physique qui existe en baie de Seine (courants de marée, stratification haline, courants de densité) et de modéliser le continuum fleuve-estuaire-baie, un modèle hydrodynamique tri-dimensionnel (SiAM-3D) a été développé (Cugier et Le Hir, 2002). A ce modèle ont été couplés un module sédimentaire et un module écologique identique à celui développé dans les modélisations compartimentales précédemment citées. Les résultats obtenus grâce à ce modèle 3D sont nombreux ( $\mathrm{Cu}$ gier, 1999) et ne peuvent être tous détaillés ici. Les conclusions concer- nant le rôle limitant des différents sels nutritifs déduites des modélisations précédentes ne sont pas remises en cause par le raffinement physique. La modélisation 3D permet toutefois une meilleure représentation et localisation des structures physiques (panache) et biologiques (bloom phytoplanctonique).

Après quelques exemples de validation du modèle, nous nous sommes intéressés dans ce document à deux résultats principaux. Le premier concerne la répartition du maximum des flagellés dans le panache, qui diffère selon les années. Ce phénomène semble pouvoir être expliqué par la qualité des apports en sels nutritifs différente certaines années. Le second résultat est une utilisation du modèle pour tenter de répondre à la question de savoir comment réagirait l'écosystème à une diminution plus ou moins forte des apports en azote de la Seine. Différents scénarios de réduction ont ainsi été réalisés et l'impact en terme de réduction de la production phytoplanctonique analysé.

\section{DESCRIPTION DU MODĖLE}

\subsection{Le modèle hydrosédimentaire}

La baie de Seine est un milieu où l'hydrodynamique est complexe et fortement influencée par la marée. Les forts gradients verticaux de densité observés en baie de Seine orientale, conferent une structure tridimensionnelle à la masse d'eau et 
influencent fortement la circulation (panache de surface, circulation résiduelle). Ainsi, afin de bien représenter ces phénomènes, un modèle hydrodynamique tridimensionnel (SiAM-3D; Cugier et Le Hir, 2002) a été développé. Le modèle 3D prend en compte le fond sédimentaire avec une ou plusieurs couches de sédiments. II gère les phénomènes d'érosion/dépôt, ainsi que les processus de tassement du matériel particulaire, et cela pour plusieurs classes de particules si nécessaire. Le modèle permet la résolution des équations sur un maillage horizontal rectiligne orthogonal à mailles inégales (fig. 3a) qui donne la possibilité d'un raffinement plus important dans certaines zones du domaine d'étude (zones à forts gradients horizontaux comme les estuaires par exemple). Sur la verticale, il utilise un système de coordonnées réelles, permettant le découpage en couches fixes d'épaisseurs quelconques, avec positionnement exact du fond et de la surface libre (fig. 3b). Le modèle gère l'apparition et la disparition de couches en surface générées par les variations du niveau (marée, vent). Le maillage horizontal irrégulier permet une prise en compte originale des fleuves ou rivières respectant la bathymétrie et les sections d'écoulement sans une augmentation trop importante du nombre de mailles. La Seine est représentée jusqu'au barrage de Poses, la limite amont de propagation de la marée, à $150 \mathrm{~km}$ environ de l'embouchure.

\subsection{Le modèle écologique}

Le modèle hydrosédimentaire SiAM-3D est couplé à un modèle écologique (Cugier, 1999) déjà utilisé dans une modélisation compartimentale de la baie de Seine (Guillaud et Ménesguen, 1998). Le schéma conceptuel du modèle est illustré par la figure 4.

Les trois éléments nutritifs (azote, phosphore et silicium) sont pris en compte sous leur forme inorganique dissoute et sous leur forme détritique. Le phosphore adsorbé sur les particules est également considéré. Deux catégories de phytoplancton sont modélisées: les diatomées, catégorie majoritaire en terme de biomasse dans le milieu, qui ont pour particularité d'utiliser la silice pour former leur frustule; les flagellés, dont certaines espèces toxiques apparaissent en baie de Seine régulièrement (Dinophysis cf. acuminata), provoquant des périodes d'interdiction de ramassage et de commercialisation des coquillages. Enfin une variable "zooplancton " est utilisée comme facteur de contrôle de la production phytoplanctonique par le broutage. L'ensemble de ces compartiments interagissent et sont reliés entre eux par un certain nombre de processus schématisés par des flèches (consommation, minéralisation, mortalité, broutage, ....). Le modèle a permis de simuler 22 années (1976-1997) en prenant pour chacune d'entre elles les données météorologiques Météo-France au Havre (Cap de la Hève) et les apports 

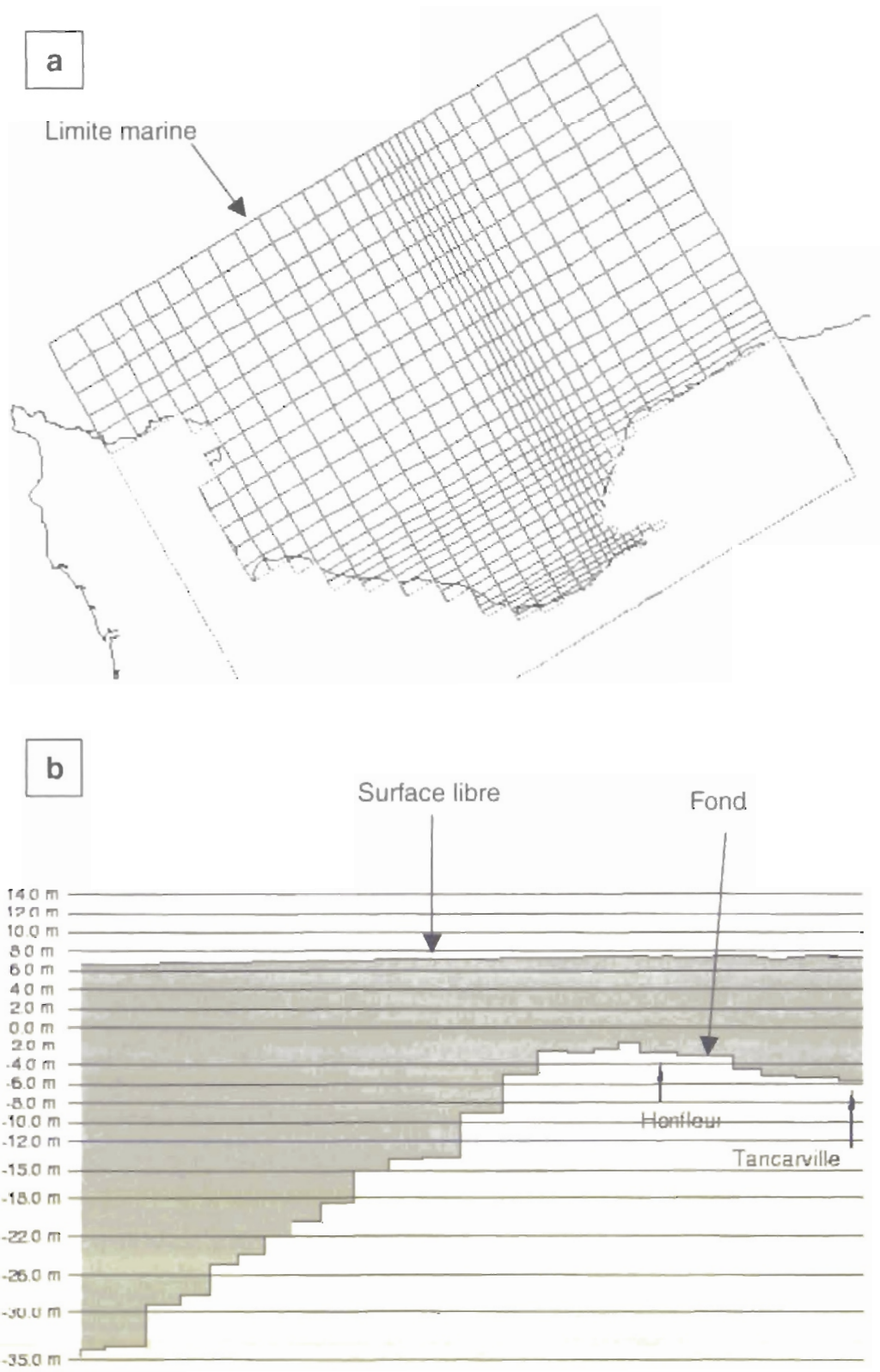

Fig. 3. - Implantation du maillage en baie de Seine. - maillage horizontal (a) et vertical (b) (L'estuaire amont de la Seine n'est pas représenté).

Fig 3. - Computation grid position in the baie de Seine. - Horizontal (a) and vertical (b) grid (The upper Seine estuary is not represented). 
Modélisation écologique tridimensionnelle (3D) de la baie de Seine (Manche, France)

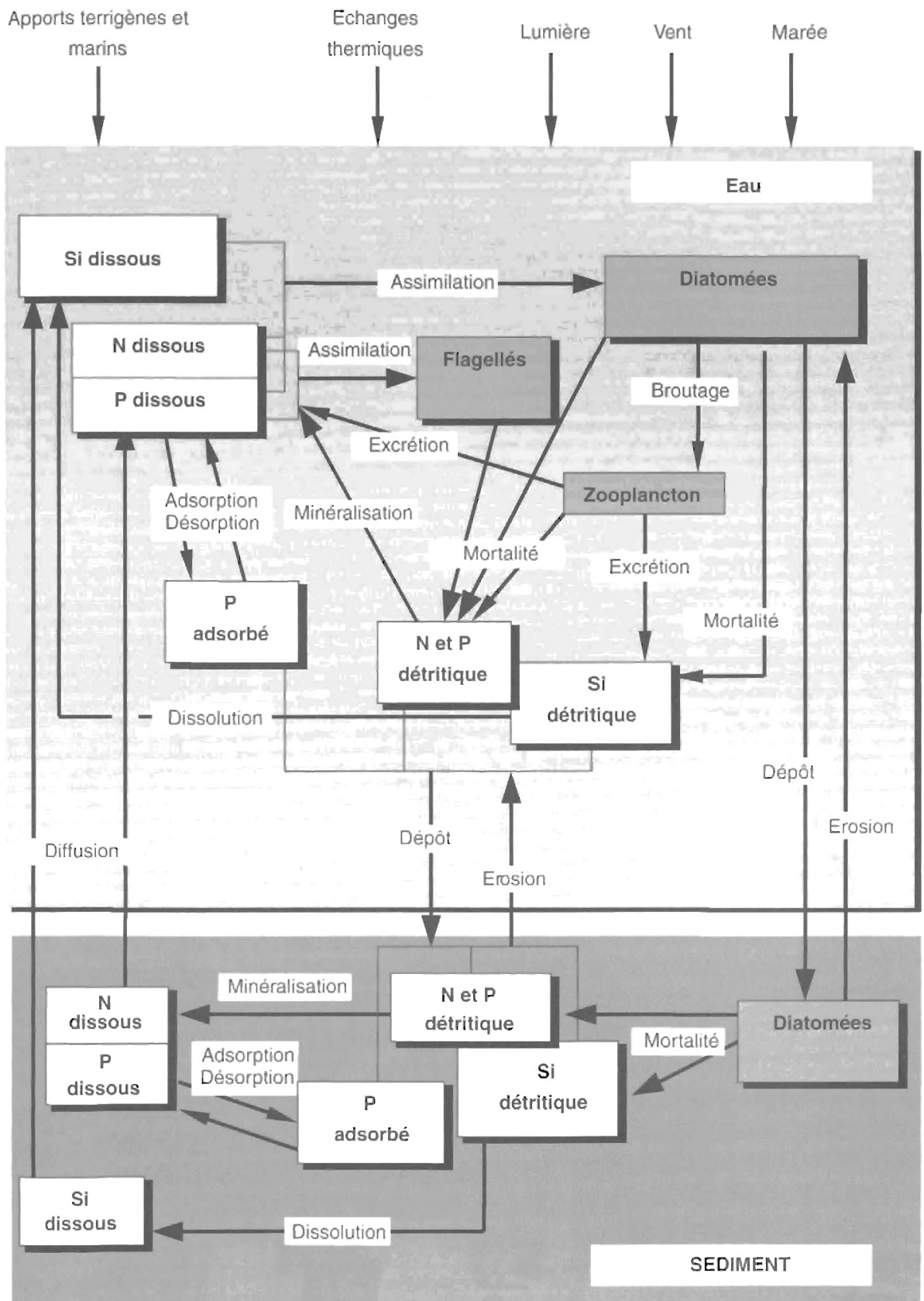

Fig. 4. - Schéma conceptuel du modèle écologique.

Fig. 4. - Conceptual scheme of the ecological model. 
mesurés en amont de la Seine (Poses) ainsi que des rejets secondaires (Orne, Vire et Douve).

\section{RÉSULTATS}

\subsection{Le modèle hydrosédimentaire}

Le modèle $3 \mathrm{D}$ reproduit convenablement les principales caractéristiques physiques de l'écosystème. II simule correctement la circulation générale en baie de Seine et la structure du panache de la Seine (Cugier et Le Hir, 2002).

La bonne reproduction des matières en suspension est un élément essentiel pour le modèle écologique car la production primaire est dépendante de l'intensité lumineuse disponible, et donc de la turbidité du milieu. La prise en compte du sédiment a permis de reproduire de manière réaliste les turbidités dans l'estuaire et le panache (Cugier et Le Hir, 2000). La figure 5 illustre la distribution des matières en suspension en surface calculées par le modèle, et met en évidence un maximum de turbidité (bouchon vaseux) dans l'estuaire.

\subsection{Le modèle écologique}

Le modèle a été utilisé pour simuler 22 années (1976-1997) et a donc permis d'étudier l'évolution de l'écosystème sur une longue période.

Les mesures du Réseau National d'Observation (C) RNO-MATE / IFREMER, banque Quadrige) entre

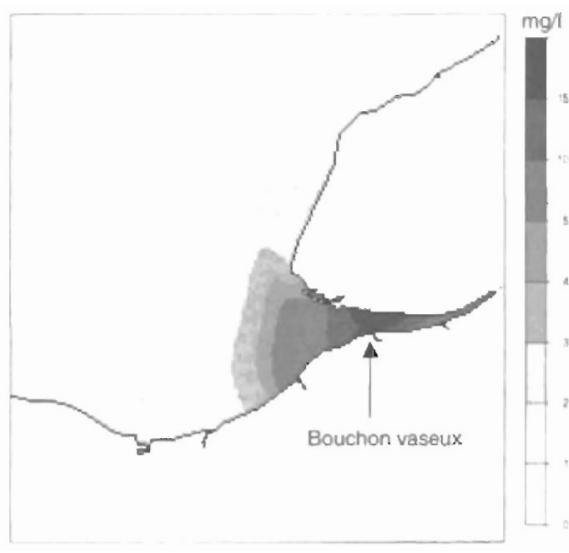

Fig. 5. - Exemple de distribution de matières en suspension simulée en surface (marée de vive eau, $3 \mathrm{~h}$ après la pleine mer).

Fig. 5. - Distribution of simulated surface suspended matter concentration (spring tide, 3 hours after high water).

1976 et 1984 ont été utilisées pour valider les résultats du modèle écologique. Les résultats du modèle ont été comparés aux mesures disponibles d'azote minéral, de phosphore, de silicium et de chlorophylle a (fig. 6) réalisées au point 2 du R.N.O. en Baie de Seine, à la sortie de l'estuaire, dans la zone du panache où les gradients de concentration sont forts. Afin de s'affranchir des variations de concentration importantes au cours du cycle de marée, les valeurs maximales et minimales des concentrations simulées durant ce cycle ont été représentées pour faciliter la comparaison avec les mesures. On obtient ainsi l'enveloppe de variation des concentrations calculées au cours du temps. 


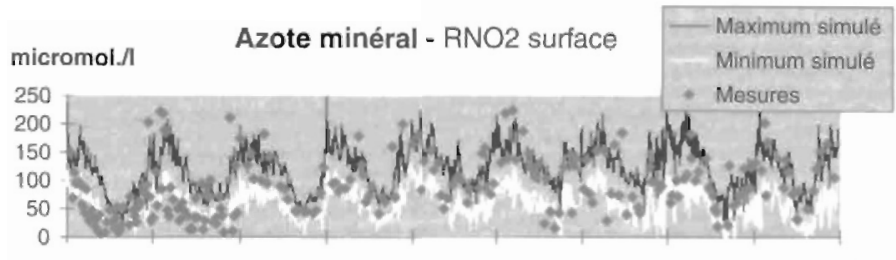

janv-76 janv-77 janv-78 janv-79 janv-80 janv-81 janv-82 janv-83 janv-84 janv-85

Dates
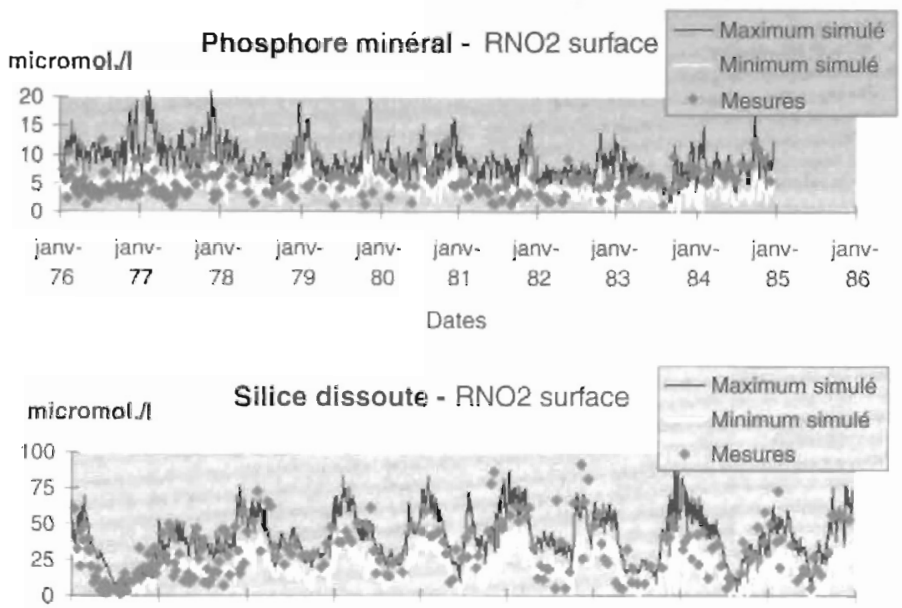

janv-76 janv-77 janv-78 janv-79 janv-80 janv-81 janv-82 janv-83 janv-84 janv-85

Dates

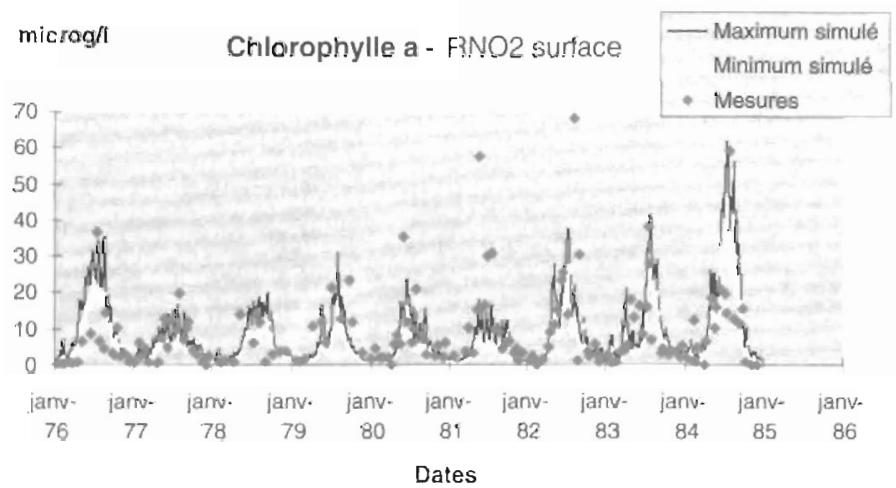

Fig. 6. - Comparaisons des concentrations simulées (trait) et mesurées (croix) pour l'azote minéral dissous, le silicale, le phosphate et la chlorophylle a en surface au point numéro 2 du Réseau National d'Observation en baie de Seine.

Fig. 6. - Comparisons between simulated (line) and measured (cross) surface concentrations for dissolved inorganic nitrogen, silicate, phosphate and chlorophyll a, at station 2 of the French National Monitoring Network in the baie de Seine. 
L'azote et le silicium montrent de fortes variations au cours de l'année, liées d'une part à la consommation par le phytoplancton, et d'autre part aux variations saisonnières des apports par la Seine. Le phosphore a, quant à lui, un cycle saisonnier moins marqué du fait de la relative constance des apports pendant l'année, et seule la consommation par le phytoplancton conduit à une légère diminution des concentrations durant la période productive. L'azote, et surtout le silicium, atteignent certaines années des niveaux très bas en période estivale. Le silicium, consommé par les diatomées, devient alors en général limitant pour la croissance de ces dernières. La comparaison avec les mesures montre une bonne capacité du modèle à reproduire les variations saisonnières et les niveaux de concentration des éléments nutritifs sur l'ensemble des 9 années.

Les variations saisonnières de la chlorophylle a sont globalement bien reproduites par le modèle tant en surface qu'au fond. Les valeurs maximales mesurées lors des blooms sont en général atteintes par la simulation à l'exception des années 1981 et 1982 où le modèle les sous-estime.

Après validation, le modèle a été utilisé pour étudier et essayer de mieux comprendre le fonctionnement de l'écosystème. Un des avantages majeurs des modèles est de pouvoir fournir des résultats synthétiques sur le fonctionnement de l'écosystème, difficilement mesurables par ailleurs. Ainsi, le maximum absolu sur l'année des concentrations phytoplanctoniques peut être facilement représenté.

Pour les diatomées, quelle que soit l'année simulée, on observe un gradient de concentration décroissant de l'estuaire vers le large avec un maximum absolu situé sur la côte du Calvados entre Ouistreham et Honfleur (fig. 7). Ce maximum varie selon les années et peut régulièrement dépasser $60 \mu \mathrm{g}$ I ' de chlorophylle a.

Pour les flagellés, deux situations sont apparues dans les 22 années simulées: soit un développement généralisé à toute la partie Est de la baie, avec un maximum le long de la côte du Calvados (fig. 8a); soit un développement limité à la zone nord du panache de la Seine face à Antifer (fig. 8b).

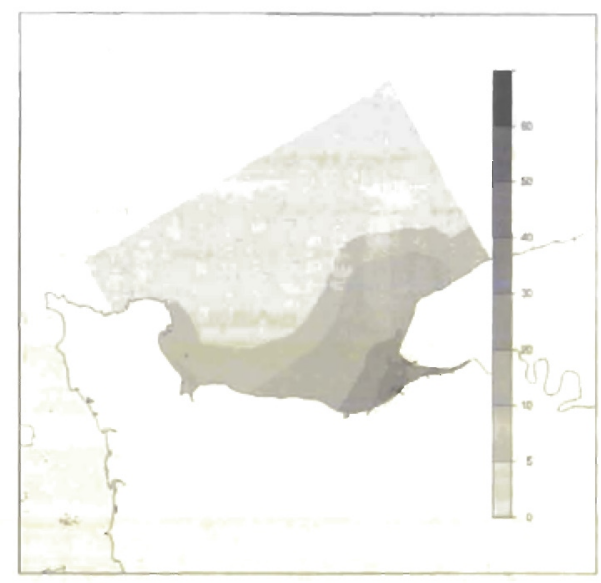

Fig. 7. - Maximum absolu de diatomées ( $\mu \mathrm{g} \mathrm{I}^{-1}$ de chlorophylle a) simulé pour l'année 1995. Fig. 7. - Distribution of simulated maximum for diatoms ( $\mu \mathrm{g} \mathrm{I}^{-1}$ of chlorophyll a) in 1995. 


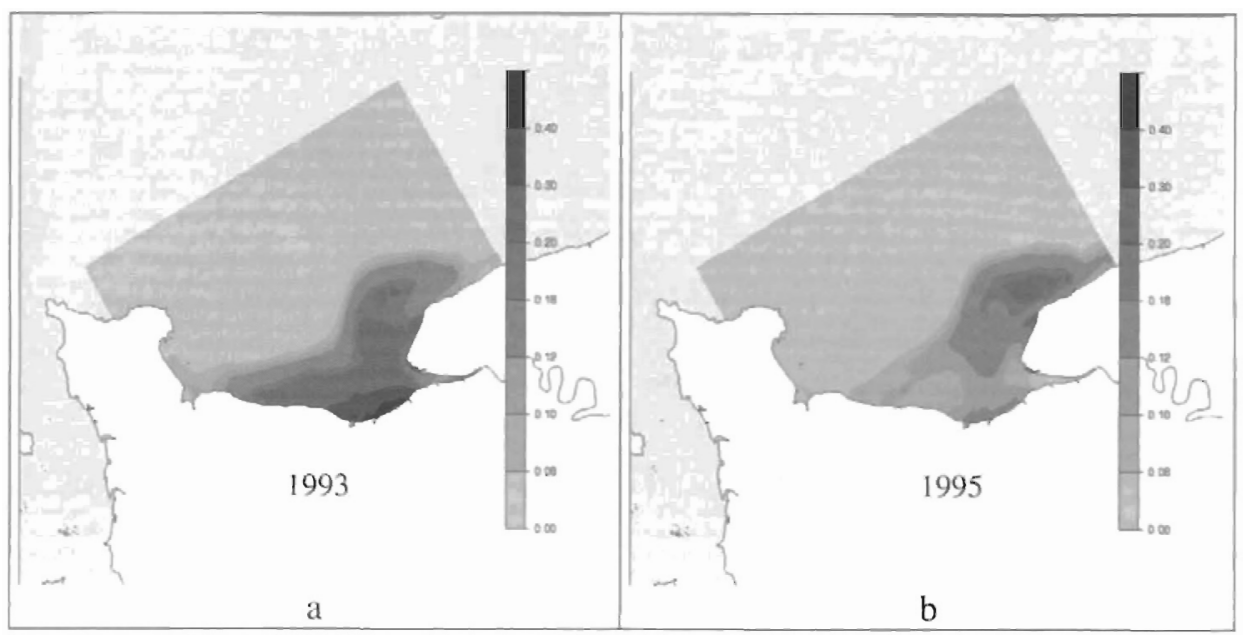

Fig. 8. - Maximum absolu de dinoflagellés ( $\mu$ mol $~^{-1}$ d'azote) simulé pour les années 1993 (a) et 1995 (b).

Fig. 8. - Distribution of simulated maximum for flagellates (umol ${ }^{-1}$ of nitrogen) in 1993 (a) and 1995 (b).

La qualité des apports en sels nutritifs par la Seine pendant la période estivale (période de production des flagellés) semble pouvoir expliquer l'apparition de l'une ou l'autre de ces situations. Les rapports moyens estivaux, silicium sur phosphore (Si/P) et silicium sur azote (Si/N) dans les apports de la Seine sont représentés pour les 22 années simulées (fig. 9); il ressort que les années où ces rapports (ou au moins l'un des deux) sont faibles correspondent à celles où les flagellés se développent de manière importante sur la côte du Calvados (barres noires).

Ainsi, la qualité des apports semble être un élément déterminant pour le développement des flagellés au sud du panache. Les années où des étiages sévères sont observés correspondent également à celles où les rapports $\mathrm{Si} / \mathrm{N}$ et $\mathrm{Si} / \mathrm{P}$ sont plus faibles, le modèle les identifiant donc comme des années où les flagellés se développent plus fortement au sud. Le cas extrême des 22 années simulées est celui de l'année 1990 où les débits estivaux de la Seine ont été les plus faibles avec des minimums inférieurs à $100 \mathrm{~m}^{3} / \mathrm{s}$. Ces années présentent un déficit accru de silice limitant la croissance des diatomées et permettant une plus grande disponibilité de l'azote et du phosphore pour les flagellés.

L'influence du rapport Si/N sur l'accroissement des populations de flagellés a déjà été évoquée par différents auteurs. Ainsi, Radach (1992) a observé que la diminution du rapport $\mathrm{Si} / \mathrm{N}$ dans les eaux côtières de mer du 
Rapport Si/N des apports moyens estivaux de la Seine

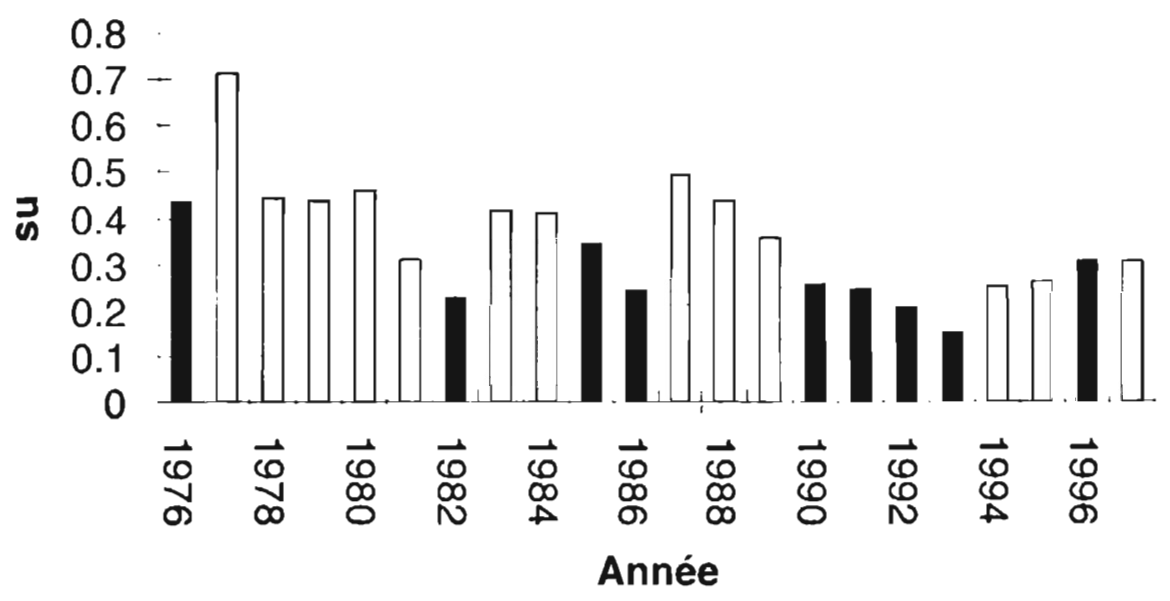

Rapport Si/P des apports moyens estivaux de la Seine

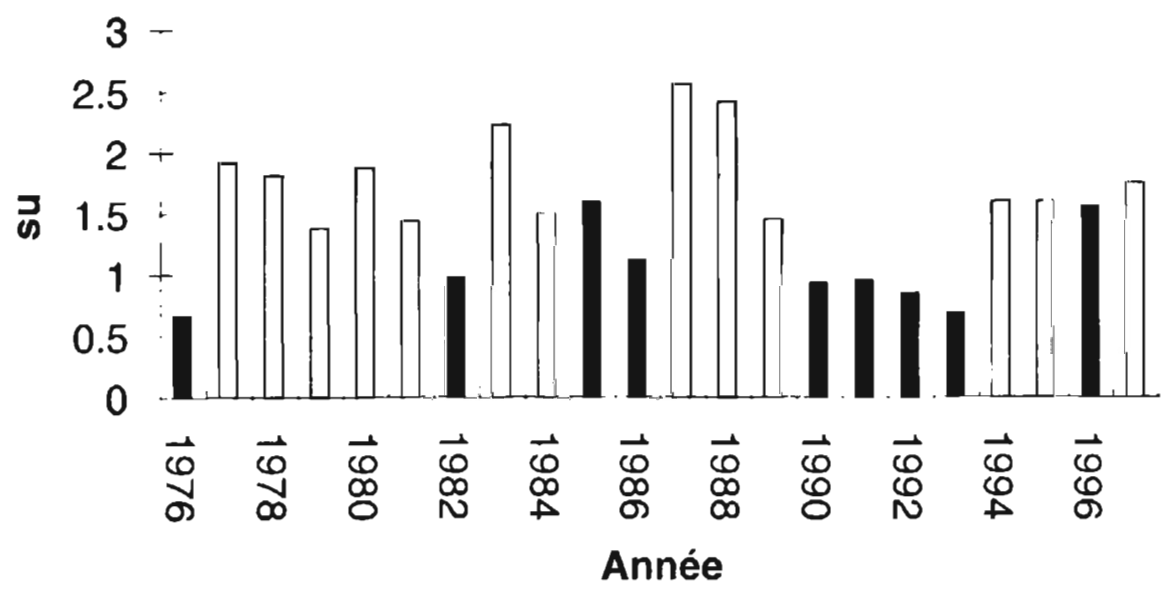

Fig. 9. - Rapports Si/N et Si/P dans les apports estivaux de la Seine entre 1976 et 1997. Les barres noires identifient les années où le modèle simule de fortes concentrations en flagellés sur la côte du Calvados.

Fig. 9. - Si/N and Si/P ratios in summer Seine river loadings from 1976 to 1997. Black bars represent years where high flagellate concentrations are simulated along the Calvados coast. 
Nord a entraîné un accroissement des flagellés par rapport aux diatomées entre 1962 et 1984. De la même manière, des expériences réalisées en mésocosmes par Schollhorn et Granéli (1993); Escaravage et al. (1995) et Sommer (1995) ont permis de mettre en évidence que la diminution du rapport $\mathrm{Si} / \mathrm{N}$ dans le milieu tend à faire augmenter le rapport flagellés/diatomées.

Dans un écosystème côtier enrichi comme la baie de Seine, où se développent de fortes biomasses algales, la question de savoir comment évoluerait l'écosystème en réponse à une réduction des apports est d'importance, d'une part sur le plan scientifique, en vue d'une meilleure compréhension du fonctionnement du système, et d'autre part dans une perspective de gestion du bassin versant. Le modèle s'avère être le seul moyen pour tenter d'apporter une réponse à ce genre de question, et une de ses applications importantes a été l'étude de scénarios de réduction du flux d'azote par la Seine, et l'estimation de l'impact sur la production phytoplanctonique. Des réductions de $10,25,50,75$ et $90 \%$ des apports d'azote minéral dissous par la Seine ont été simulées et l'impact en terme de variation de la production des diatomées et des flagellés dans le panache a été étudié. L'année de référence choisie est 1995 car elle représente une situation moyenne en terme de concentrations phytoplanctoniques (diatomées et flagellés) et de conditions environnementales (apports fluviaux, météo). Seuls les apports en azote minérale dissous de la Seine sont modifiés, tous les autres apports correspondent aux apports réels mesurés.

La figure 10 représente le pourcentage de diminution de la production des diatomées et des flagellés en fonction du pourcentage de réduction des apports d'azote.

La réduction des flagellés est nettement plus importante que celle des diatomées. Le taux maximal d'abattement de la production des flagellés est proche de $60 \%$. Les diatomées, qui représentent la majeure partie de la biomasse phytoplanctonique, ont une réaction nettement moins importante et la réduction maximale de leur production dépasse à peine $20 \%$. Pour les faibles taux de réduction d'apport ( $\leq 25 \%$ ), l'impact sur les diatomées est quasi nul ; ceci peut

\section{Réduction des apports d'azote par la Seine}

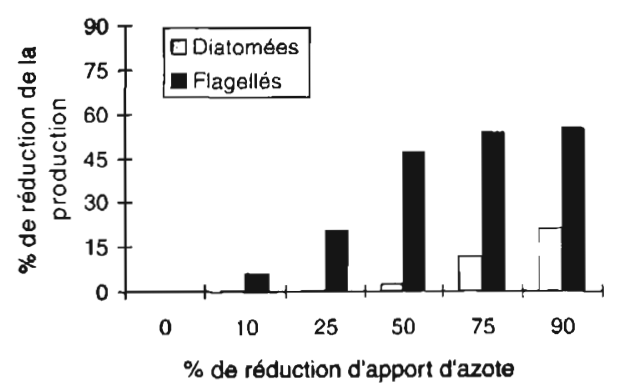

Fig. 10. - Impact de la réduction des apports d'azote minéral dissous par la Seine en terme de réduction de la production phytoplanctonique.

Fig. 10. - Impact of the reduction of dissolved inorganic nitrogen loadings from the Seine river on phytoplanktonic production. 
s'expliquer par le fait que les apports d'azote par la Seine se faisant en très grandes quantités, une réduction modeste de ces apports n'est pas suffisante pour provoquer un effet de limitation lié à l'azote.

Par contre, il peut être surprenant qu'une réduction de $90 \%$ des apports d'azote minéral par la Seine ne conduise qu'à une réduction de $50 \%$ de la production des flagellés mais surtout à une réduction d'à peine $20 \%$ de la production des diatomées. II faut cependant noter que les apports par la Seine en azote détritique (5 à $10 \%$ des apports d'azote total) n'ont pas été réduits et que, par le biais du processus de reminéralisation, ils sont une source d'azote minéral pour le système. D'autre part, il existe d'autres sources d'azote prises en compte par le modèle : ce sont les rivières secondaires de la baie de Seine et notamment l'Orne, mais aussi, et surtout, les apports provenant du large qui, quoique faibles en concentration, représentent des masses d'azote importantes, étant donnés les volumes considérés. Ces apports par le large en azote minéral total ont été estimés dans une précédente étude par modélisation à 140000 t/an sur l'ensemble de la baie de Seine (Guillaud et Ménesguen, 1998). Ceci est du même ordre de grandeur que les apports d'azote minéral d'origine terrestre (de 50000 à 160000 t/an). L'essentiel des apports en azote du large se fait par la partie nord-ouest de la baie, au niveau du Cotentin. Une des perspective d'utilisation du modèle serait de pouvoir quantifier plus précisément la contribution de chacun des apports à la production phytoplanctonique.

\section{CONCLUSIONS}

La modélisation tri-dimensionnelle reproduit les principales caractéristiques tant physiques que biologiques de l'écosystème de la baie de Seine.

L'analyse des résultats des simulations a permis de mieux comprendre la dynamique du compartiment des flagellés et notamment le rôle des apports en sels nutritifs par la Seine sur la plus ou moins grande présence de ceux-ci sur la côte du Calvados. Ainsi, il semble que les années où les rapports estivaux $\mathrm{Si} / \mathrm{N}$ et/ou Si/P sont faibles soient favorables au développement des flagellés. La carence en silicium limite alors la croissance des diatomées et permet une plus grande disponibilité de l'azote et du phosphore pour les flagellés. Les années sèches sont typiquement des années où la concentration en silicium est plus faible et donc où les flagellés se développent plus facilement.

L'étude des différents scénarios de réduction des apports d'azote minéral par la Seine montre un impact nettement plus fort sur la production des flagellés que sur celle des diatomées. Une réduction de $90 \%$ des apports d'azote minéral conduit en effet à une réduction d'environ $60 \%$ de la production des flagellés alors que celle des diatomées atteint à peine $20 \%$. Ce faible impact mettrait en évidence 
la présence d'autres sources d'azote minéral qui permettraient de soutenir la production. Ainsi, la matière organique, via les processus de minéralisation, joue sans doute un rôle important. Les autres apports directs par les rejets secondaires en baie de Seine (Orne) et en particulier par le large peuvent également contribuer à la production. Une perspective d'utilisation de ce modèle serait de "tracer " ces différentes sources potentielles afin d'estimer leur poids respectif dans la production phytoplanctonique.

\section{RÉFÉRENCES BIBLIOGRAPHIQUES}

Cugier, P., 1999. Modélisation du devenir à moyen terme dans l'eau et le sédiment des éléments majeurs ( $N, P, S i$, O) rejetés par la Seine en baie de Seine. Thèse de doctorat de l'université de Caen, $241 \mathrm{p}$.

Cugier, P., Le Hir, P., 2000. Modélisation $3 D$ des matières en suspension en baie de Seine Orientale (Manche, France). C. R. Acad. Sci. Paris, Sciences de la Terre et des planètes, 331 . 287-294.

Cugier, P., Le Hir, P., 2002. Development of a 3D hydrodynamical model for coastal ecosystem modelling. Application to the plume of the Seine River (France). Estuar. Coast. Shelf Sc., 55, 673-695.

Escaravage, V., Prins, T.C., Nijdam, C., Smaal, A.C., Peeters, J.C.H., 1995.
The response of phytoplankton communities to $\mathrm{N}$-input reduction results from mesocosm experiments. ICES Annual Science Conference; C. M. 1995/T :10. $16 \mathrm{p}$.

Guillaud, J.F., 1993. Les apports en sels nutritifs dans l'estuaire de la Seine. IFREMER, Rapport interne DEL/93.04/ BREST, $32 p$.

Guillaud, J.F., Ménesguen, A., 1998. Modélisation sur vingt ans (1976-1995) de la production phytoplanctonique en baie de Seine (France). Oceanol. Acta, 21 (6), 887-906.

Guillaud, J.F., Andrieux, F., Ménesguen, A., 2000. Biogeochemical modelling in the Bay of Seine (France) : an improvement by introducing phosphorus in nutrient cycles. J. Mar. Syst., 25, 369-386.

Ménesguen, A., Guillaud, J.F., Aminot, A., Hoch, T., 1995. Modelling the eutrophication in a river plume : the Seine river case study (France). Ophelia, 42, 205-225.

Radach, G., 1992. Ecosystem functioning in the German Bight under continental nutrient inputs by river. Estuaries, 15, 477-496.

Schollhorn, E., Graneli, E., 1993. Is the increase of flagellates in coastal waters caused by changes in ratio of N, P and $\mathrm{Si}$ ? In Toxic phytoplancton Blooms in the Sea. (Smayda T. J. and Shimizu Y. Eds), Elsevier Science Publishers B. V., 811-817.

Sommer, U., 1995. Eutrophication related changes in phytoplankton species composition: is there a role of nutrient competition? ICES Annual Science Conference; C. M. 1995/T : 7. 6 p. 